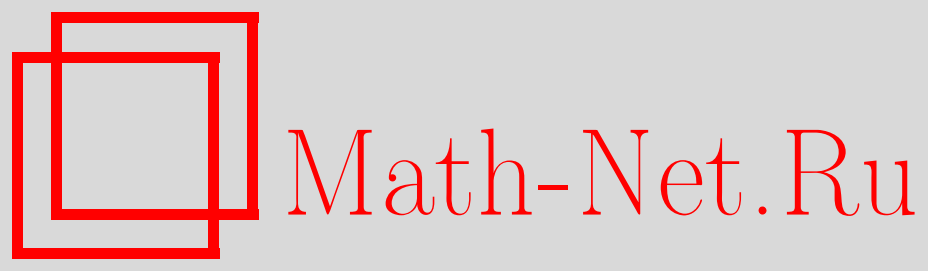

Л. Д. Фаддеев, Нулевые моды для квантовой модели Лиувилля, Функи. анализ и его прил., 2014, том 48, выпуск 3, 14-23

DOI: https://doi.org/10.4213/faa3149

Использование Общероссийского математического портала MathNet.Ru подразумевает, что вы прочитали и согласны с пользовательским соглашением

http://www . mathnet.ru/rus/agreement

Параметры загрузки:

IP : 3.85 .183 .62

26 апреля 2023 г., 05:05:40

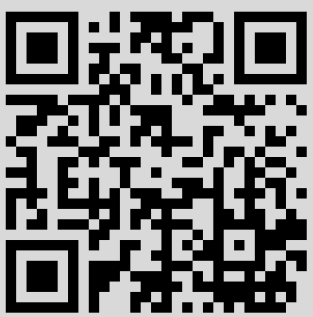


Функционалъный анализ и его приложения

2014, т. 48, вып. 3, с. 14-23

УДК 517.9

\title{
Нулевые моды для квантовой модели Лиувилля*
}

\author{
(C) 2014. Л. Д. ФАДДЕЕВ
}

К столетию со дня рождения И. М. Гельфанда

Обсуждается проблема определения нулевых мод для квантовой модели Лиувилля и описывается их представление в гильбертовом пространстве.

\section{§1. Введение и постановка задачи}

Квантовая модель Лиувилля является базисным примером конформной теории поля. Она представляет собой квантование динамической системы для вещественного поля $\phi(x, t)$, заданной на цилиндре $(x, t) \in \mathbb{S}^{1} \times \mathbb{R}$ гамильтонианом

$$
H=\frac{1}{2 \gamma} \int_{0}^{2 \pi}\left(\pi^{2}+\phi_{x}^{2}+e^{2 \phi}\right) d x
$$

и канонической скобкой Пуассона

$$
\{\phi(x), \phi(y)\}=0, \quad\{\pi(x), \pi(y)\}=0, \quad\{\pi(x), \phi(y)\}=\gamma \delta(x-y)
$$

в терминах начальных данных $\phi(x)=\phi(x, 0), \pi(x)=\phi_{t}(x, 0)$. Соответствующее уравнение движения имеет вид

$$
\phi_{t t}-\phi_{x x}+e^{2 \phi}=0 .
$$

В основе квантового подхода, развитого рядом авторов (см. обзор [1] и цитированную там литературу), лежит классическая формула Лиувилля

$$
e^{2 \phi}=-4 \frac{f^{\prime}(x-t) g^{\prime}(x+t)}{(f(x-t)-g(x+t))^{2}}
$$

и параметризация функций $f(x)$ и $g(x)$ через свободные поля и нулевые моды типа

$$
\ln f(x)=Q-\frac{P x}{\pi}+\chi(x),
$$

где $Q$ и $P$ - канонические переменные, а периодическое поле $\chi(x)$ задается осцилляторными степенями свободы. Поле $g(x)$ параметризуется аналогично с теми же $P$ и $Q$. Возникает картина для соответствующего гильбертова пространства

$$
\mathfrak{H}=L_{2}(\mathbb{R}) \otimes \mathscr{H}_{L} \otimes \mathscr{H}_{R},
$$

где в $\mathscr{H}_{L}$ и $\mathscr{H}_{R}$ представляют осцилляторы, а $L_{2}(\mathbb{R})$ является пространством нулевых мод $(P, Q)$. Однако на некотором этапе возникает инвариантность по отношению к отражению $P \rightarrow-P$, происхождение которой не всегда ясно.

*Работа над этой статьей частично поддержана грантами РФФИ 11-01-00570-а, 11-0112037-офи_м и программой РАН «Математические проблемы нелинейной динамики» 
В данной работе, которая следует предыдущей публикации [4], я приведу аргументы для этой инвариантности и метод ее реализации в гильбертовом пространстве.

\section{§2. Классическая теория}

Уравнение (1) реализуется как соотношение нулевой кривизны

$$
\left[\widehat{L}_{1}, \widehat{L}_{0}\right]=0
$$

для связности

$$
\widehat{L}_{1}=\frac{d}{d x}-L_{1}, \quad \widehat{L}_{0}=\frac{d}{d t}-L_{0},
$$

где $2 \times 2$-матрицы $L_{1}, L_{0}$ параметризуются полем $\phi(x, t)$ :

$$
L_{1}=\frac{1}{2}\left(\begin{array}{cc}
\phi_{t} & e^{\phi} \\
e^{\phi} & -\phi_{t}
\end{array}\right), \quad L_{0}=\frac{1}{2}\left(\begin{array}{cc}
\phi_{x} & -e^{\phi} \\
e^{\phi} & -\phi_{x}
\end{array}\right) .
$$

Пусть

$$
T(x, t)=\left(\begin{array}{ll}
A(x, t) & B(x, t) \\
C(x, t) & D(x, t)
\end{array}\right)
$$

- голономия, т. е. решение совместных уравнений

$$
T_{x}=L_{1} T, \quad T_{t}=L_{0} T
$$

с начальным условием

$$
T(0,0)=I .
$$

Легко проверить, что проективные компоненты

$$
f(x, t)=\frac{A(x, t)}{B(x, t)}, \quad g(x, t)=\frac{C(x, t)}{D(x, t)}
$$

удовлетворяют уравнениям

$$
f_{x}=f_{t}, \quad g_{x}=-g_{t}
$$

и, таким образом,

$$
f(x, t)=f(x-t), \quad g(x, t)=g(x+t) .
$$

Из (4) следуют начальные условия

$$
f(0)=\infty, \quad g(0)=0
$$

и ограничения

$$
f^{\prime}(0)<0, \quad g^{\prime}(0)>0,
$$

из которых вытекает, что $f(x)$ и $g(x)$ положительны. Формула Лиувилля $(2)$ реализуется этими функциями.

Введем для окружности $t=0$ монодромию

$$
M=T(2 \pi, 0)=\left(\begin{array}{ll}
a & b \\
c & d
\end{array}\right) .
$$

Это гиперболическая унимодулярная матрица с положительными матричными элементами. Ясно, что $f$ и $g$ удовлетворяют условиям квазипериодичности:

$$
f(x+2 \pi)=\frac{a f(x)+c}{b f(x)+d}, \quad g(x+2 \pi)=\frac{a g(x)+c}{b g(x)+d} .
$$


Эти условия можно упростить, если привести монодромию к диагональному виду, взяв

$$
M N=N D,
$$

где

$$
D=\left(\begin{array}{cc}
\lambda & 0 \\
0 & 1 / \lambda
\end{array}\right), \quad N=\left(\begin{array}{cc}
1 & 1 / \xi \\
\eta & 1
\end{array}\right) .
$$

Параметр $\lambda$ дает собственное значение монодромии, а $\xi$ и $\eta-$ ее неподвижные точки, т. е. решения квадратного уравнения

$$
b \xi^{2}-(a-d) \xi-c=0, \quad a+d=\lambda+\frac{1}{\lambda} .
$$

Леон Тахтаджян и я в 1984 г. сосчитали скобки Пуассона элементов $a, b, c, d$ (опубликовано в 1986 г. в [5]):

$$
\begin{array}{rlrl}
\{a, b\}=\frac{1}{2} \gamma a b, & \{a, c\} & =\frac{1}{2} \gamma a c, \\
\{d, b\}=-\frac{1}{2} \gamma b d, & \{d, c\}=-\frac{1}{2} \gamma c d, \\
\{b, c\}=0, & \{a, d\}=\gamma b c
\end{array}
$$

(заметим, что эти соотношения легли в основу теории квантовых групп (см. [6], $[7]))$. Из этих соотношений следует, что $\lambda, \xi$ и $\eta$ можно параметризовать в виде

$$
\lambda=e^{-P}, \quad \xi=\alpha e^{Q}, \quad \eta=-\alpha e^{-Q}
$$

со скобками

$$
\{P, Q\}=\frac{\gamma}{2}, \quad\{P, \alpha\}=0, \quad\{Q, \alpha\}=0 .
$$

В терминах этих переменных монодромия $T$ приобретает вид

$$
M=\frac{1}{\operatorname{ch} Q}\left(\begin{array}{cc}
\operatorname{ch}(Q-P) & \alpha^{-1} \operatorname{sh} P \\
\alpha \operatorname{sh} P & \operatorname{ch}(Q+P)
\end{array}\right) .
$$

Теперь очевидно, что для образа

$$
\hat{f}=N(f)=\frac{f+\eta}{f / \xi+1}
$$

функции $f$ относительно преобразования Мёбиуса имеем

$$
\hat{f}(0)=\xi=\alpha e^{Q}, \quad \hat{f}(2 \pi)=e^{-2 P} \hat{f}(0),
$$

так что

$$
\ln \frac{\hat{f}(x)}{\alpha}=Q-\frac{P x}{\pi}+\chi(x),
$$

где $\chi(x)$ периодична. Мы получили формулу (3). Однако элементы матрицы $M$ положительны только при условии, что

$$
P>0 \text {. }
$$

Таким образом, фазовое пространство для нулевых мод $P$ и $Q$ представляет собой полуплоскость и его квантование следует провести корректно. Мы сделаем это после введения новых канонических переменных вместо $P$ и $Q$ и квантовой реализации соответствующего канонического преобразования. 
Введем преобразование переменных

$$
u=\frac{\operatorname{ch} Q}{\operatorname{ch}(Q-P)}=\frac{1}{a}, \quad v=\frac{\operatorname{sh}^{2} P}{\operatorname{ch} Q \operatorname{ch}(Q-P)}=\frac{b c}{a},
$$

которое отображает верхнюю полуплоскость $(P, Q)$ в положительный квадрант

$$
u>0, \quad v>0 .
$$

Скобки Пуассона для $u$ и $v$ имеют вид

$$
\{u, v\}=-\gamma u v
$$

так что их логарифмы дают канонические переменные на $\mathbb{R}^{2}$ и их квантование тривиально.

Монодромия $M$ приобретает вид

$$
M=\left(\begin{array}{cc}
u^{-1} & \alpha \sqrt{v / u} \\
\alpha^{-1} \sqrt{v / u} & u+v
\end{array}\right),
$$

и мы можем построить для нее квантовую реализацию.

\section{§3. Квантование}

Квантовым аналогом переменных $u$ и $v$ является вейлева пара $u$ и $v$ с соотношением

$$
u v=q^{2} v u, \quad q=e^{i \gamma / 2} .
$$

Переменная $\alpha$ остается центральным элементом. Чтобы быть ближе к обозначениям из теории автоморфных функций, положим

$$
\gamma=2 \pi \tau
$$

и для $\tau$ выберем параметризацию через мнимые полупериоды $\omega, \omega^{\prime}$,

$$
\tau=\frac{\omega^{\prime}}{\omega}, \quad \omega \omega^{\prime}=-\frac{1}{4},
$$

лежащие на верхней мнимой полуоси.

Операторы $u, v$ реализуются в $L_{2}(\mathbb{R})$ следующим образом:

$$
u f(x)=e^{-i \pi x / \omega} f(x), \quad v f(x)=f\left(x+2 \omega^{\prime}\right) .
$$

Это существенно самосопряженные операторы с областью определения $\mathscr{D}$, состоящей из аналитических функций вида

$$
e^{-\alpha x^{2}} e^{\beta x} P(x)
$$

где $\alpha>0, \beta \in \mathbb{C}$, а $P(x)$ - полином с комплексными коэффициентами.

Квантовый аналог монодромии $M$ после естественного упорядочивания множителей задается в виде

$$
M=\left(\begin{array}{cc}
u^{-1} & \alpha q^{-1 / 4} u^{-1 / 2} v^{1 / 2} \\
\alpha^{-1} q^{-1 / 4} u^{-1 / 2} v^{1 / 2} & u+v
\end{array}\right) .
$$

Наша первая задача - диагонализовать $\operatorname{tr} M$, т. е. оператор

$$
L=u+u^{-1}+v \text {. }
$$


Этот оператор сейчас хорошо известен, он имеет много интерпретаций. Например, в квантовой теории Тейхмюллера ([8], [9]) он называется длиной геодезической, в теории разложения тензорного произведения двух неприводимых представлений модулярного дубля квантовой теории групп $S L_{q}(2, \mathbb{R})$ он появляется как главная часть соответствующего оператора Казимира [10]. Спектральная теория для оператора $L$ исследовалась Кашаевым [11].

Оператор $L$ имеет однократный непрерывный спектр, сосредоточенный на полуоси $2<\lambda<\infty$. Соответствующие обобщенные собственные функции явно выражаются через функцию

$$
\gamma(x)=\exp \left\{-\frac{1}{4} \int_{-\infty}^{\infty} \frac{e^{i x t}}{\sin \omega t \sin \omega^{\prime} t} \frac{d t}{t}\right\}
$$

где сингулярность при $t=0$ обходится сверху. Эта функция получила большое распространение в последние 10 лет. Я предложил назвать ее квантовым дилогарифмом [12]. Подробное описание ее свойств можно найти в [13]. В приложении приводятся некоторые ее свойства, используемые в этой работе.

Основное свойство - функциональное уравнение

$$
\frac{\gamma\left(x+\omega^{\prime}\right)}{\gamma\left(x-\omega^{\prime}\right)}=1+e^{-i \pi x / \omega}
$$

позволяет утверждать, что обобщенная функция

$$
\psi(x, s)=\gamma\left(x-s-\omega^{\prime \prime}+i 0\right) \gamma\left(x+s-\omega^{\prime \prime}+i 0\right) e^{-i \pi\left(x-\omega^{\prime \prime}\right)^{2}}
$$

при $s \in \mathbb{R}$ является собственной функцией оператора $L$ с собственным значением

$$
e^{i \pi s / \omega}+e^{-i \pi s / \omega}=2 \cos \pi s / \omega .
$$

Здесь

$$
\omega^{\prime \prime}=\omega+\omega^{\prime} .
$$

Функция $\gamma\left(z-\omega^{\prime \prime}\right)$ имеет полюс при $z=0$, и слагаемое $+i 0$ характеризует его обход.

Кашаев [11] доказал свойства ортонормированности и полноты

$$
\begin{gathered}
\int \overline{\psi(x, s)} \psi\left(x, s^{\prime}\right) d x=\frac{1}{\rho(s)}\left(\delta\left(s-s^{\prime}\right)+\delta\left(s+s^{\prime}\right)\right), \\
\int_{0}^{\infty} \psi(x, s) \overline{\psi(y, s)} \rho(s) d s=\delta(x-y),
\end{gathered}
$$

где мера $\rho(s)$ имеет вид

$$
\rho(s)=-4 \sin \frac{\pi s}{\omega} \sin \frac{\pi s}{\omega^{\prime}} .
$$

Мы делаем естественный вывод: гильбертово пространство для диагонального представления оператора $P$ - это $L_{2}\left(\mathbb{R}_{+}, \rho\right)$.

Посмотрим теперь, как выглядит недиагональный элемент матрицы $M$ в этом представлении. Обозначим через $U$ интегральный оператор с ядром $\psi(x, s)$, связывающий пространства $L_{2}\left(\mathbb{R}_{+}, \rho\right)$ и $L_{2}(\mathbb{R})$. Пусть $r$ и $Z$ задают вейлеву пару в пространстве функций $F(s)$ на всей оси $-\infty<s<\infty$ (в дальнейшем нам надо следить, какие комбинации $r$ и $Z$ имеют смысл в $\left.L_{2}\left(\mathbb{R}_{+}, \rho\right)\right)$ :

$$
r F(s)=F\left(s+\omega^{\prime}\right), \quad Z f=e^{i \pi s / \omega} F(s) .
$$


Из функционального уравнения (7) получаем, что интегральные операторы $v^{1 / 2} U r$ и $v^{1 / 2} U r^{-1}$ имеют ядра

$$
\begin{aligned}
& \psi\left(x+\omega^{\prime}, s-\omega^{\prime}\right)=\left(1-\frac{u}{Z}\right) \frac{1}{i} q^{-1 / 2} u^{-1 / 2} \psi(x, s), \\
& \psi\left(x+\omega^{\prime}, s+\omega^{\prime}\right)=(1-u Z) \frac{1}{i} q^{-1 / 2} u^{-1 / 2} \psi(x, s),
\end{aligned}
$$

откуда

$$
q^{1 / 4} u^{-1 / 2} v^{1 / 2} U=U \frac{1}{i}\left(Z-Z^{-1}\right)\left(r-r^{-1}\right)^{-1} .
$$

Оператор в правой части имеет смысл в пространстве четных функций и самосопряжен в $L_{2}\left(\mathbb{R}_{+}, \rho\right)$.

Итак, мы получили новое представление для элементов матрицы монодромии:

$$
U^{-1}(a+d) U=Z+Z^{-1}, \quad U^{-1} b U=\frac{\alpha}{i}\left(Z-Z^{-1}\right) \frac{1}{r-r^{-1}} .
$$

Поучительно сравнить эти квантовые формулы с классическими из (6):

$$
a+d=2 \operatorname{ch} P, \quad b=\alpha \frac{\operatorname{sh} P}{\operatorname{ch} Q} .
$$

Естественно отождествить $Z$ с $e^{P}$, a $r^{-1}$ с $e^{Q}$, однако $\operatorname{ch} Q$ в классическом случае заменяется на $i \operatorname{sh} Q$, что соответствует сдвигу $Q$ на $i \pi / 2$. Решить аналог квадратного уравнения (5) для нахождения представления операторов $u$ и $v$ мне не удалось. Впрочем, для этой работы это не нужно.

\section{§4. Коэффициент отражения}

Естественно желание избавиться от меры $\rho(s)$ и найти реализацию операторов $P$ и $b$ в обычном пространстве $L_{2}(\mathbb{R})$. Ясно, что эта реализация должна получиться только в подпространстве. Мы покажем, что можно построить соответствующий проектор в виде

$$
\Pi=\frac{1}{2}(I+K S)
$$

где $K$ - оператор отражения,

$$
K F(s)=F(-s),
$$

а оператор $S$ является умножением на множитель $S(s)$, такой, что

$$
\overline{S(s)}=S(-s)=S^{-1}(s) .
$$

Этот множитель естественно назвать коэффициентом отражения.

Для вывода заметим, что меру $\rho(s)$ можно факторизовать в виде

$$
1 / \rho(s)=M(s) M(-s),
$$

где

$$
M(s)=\text { const } e^{-2 i \pi s\left(s-\omega^{\prime \prime}\right)} \gamma\left(2 s-\omega^{\prime \prime}\right) ;
$$

здесь const можно выбрать так, что выполняется соотношение

$$
\overline{M(s)}=M(-s),
$$


если использовать соотношения (11), (13) из приложения. Явный вид этого множителя не будет существенным для нас в дальнейшем.

Из функционального уравнения (7) следует, что

$$
\begin{aligned}
& M\left(s+\omega^{\prime}\right)=M(s) \frac{1}{i}\left(Z-Z^{-1}\right), \\
& M\left(s-\omega^{\prime}\right)=M(s) i\left(q^{-1} Z-q Z^{-1}\right)^{-1} .
\end{aligned}
$$

Введем оператор

$$
V=U \frac{1}{M(s)} .
$$

Его образ состоит из функций $F(s)$, удовлетворяющих уравнению

$$
F(-s)=S(s) F(s) \text {, }
$$

где

$$
S(s)=\frac{M(s)}{M(-s)},
$$

и, таким образом, лежащих в подпространстве

$$
\Pi L_{2}(\mathbb{R}),
$$

которое и является естественным гильбертовым пространством для нулевой моды $P$.

Оператор $b^{-1}=q^{1 / 4} u^{1 / 2} v^{-1 / 2}$ проносится через $V$ при использовании соотношений $(9),(10)$ :

$$
b^{-1} V=U\left(r-r^{-1}\right)\left(Z-Z^{-1}\right)^{-1} \frac{1}{M(s)}=V\left(r^{-1}+\frac{1}{Z-Z^{-1}} r \frac{1}{Z-Z^{-1}}\right) .
$$

Именно эта формула была предложена в [4] из квазиклассических соображений. Здесь мы дали ее полный квантовый вывод.

Итак, мы построили естественное квантовое пространство для нулевых мод $P$ и $Q$. Заметим, что в других работах, [14], [15], предложена другая реализация гильбертова пространства для нулевых мод и коэффициент отражения выражается через классическую Г-функцию. Факторизация типа (8) меры $\rho(s)$ имеет смысл для этого коэффициента отражения. Однако вид оператора $Q$ остается неопределенным. Было бы интересно установить более тесный контакт между этими подходами.

\section{§5. Эволюция}

В работе [4] показано, что при дискретном сдвиге времени

$$
t \rightarrow t+\pi
$$

нулевые моды $P$ и $Q$ преобразуются простым образом:

$$
P \rightarrow P, \quad Q \rightarrow P+Q .
$$

Это связано с тем, что дискретная эволюция функций $\hat{f}$ и $\hat{g}$ соответствует левому и правому движениям, при которых осцилляторные компоненты не меняются.

В терминах $u$ и $v$ эта эволюция выглядит следующим образом:

$$
u \rightarrow u+v, \quad v \rightarrow u^{-1} v(u+v)^{-1},
$$


и мы будем считать, что это верно и в квантовом случае с указанием порядка множителей. Найдем соответствующий оператор эволюции $K$ :

$$
K^{-1} u K=u+v, \quad K^{-1} v K=u^{-1} v(u+v)^{-1} .
$$

Проще вычислить оператор $K^{-1}$ :

$$
(u+v) K^{-1}=K^{-1} u, \quad K u^{-1} v K^{-1}=K^{-1} v u .
$$

Этот порядок множителей имеет смысл и в квантовом случае.

Используя функциональное уравнение (7), нетрудно показать, что унитарный оператор $K^{-1}$ имеет ядро

$$
K^{-1}(x, y)=e^{2 \pi i x y} \gamma\left(x-y-\omega^{\prime \prime}\right) .
$$

Покажем, что $\psi(x, s)$ является собственной функцией оператора $K$. Имеем $K^{-1} \psi=\int e^{2 \pi i x y} \gamma\left(x-y-\omega^{\prime \prime}\right) \gamma\left(y+s-\omega^{\prime \prime}\right) \gamma\left(y-s-\omega^{\prime \prime}\right) e^{-i \pi\left(y-\omega^{\prime \prime}\right)^{2}} d y=I(x, s)$.

Интеграл в правой части берется с использованием одного из вариантов так называемого $\beta$-интеграла (см., например, [13]).

Начнем с того, что по свойству отражения функцию $\psi(x, s)$ можно переписать в виде

где

$$
\psi(x, s)=c(s) \frac{\gamma\left(x+s-\omega^{\prime \prime}\right)}{\gamma\left(s-x+\omega^{\prime \prime}\right)} e^{2 \pi i s x},
$$

$$
c(s)=\beta e^{-i \pi s^{2}} .
$$

Теперь используем интегральное представление (15) (см. приложение):

$$
\frac{\gamma\left(x-y-\omega^{\prime \prime}\right)}{\gamma\left(s-y+\omega^{\prime \prime}\right)}=\frac{c}{\gamma\left(s-x+\omega^{\prime \prime}\right)} \int \frac{\gamma\left(s-x+\omega^{\prime \prime}+z\right)}{\gamma\left(z+\omega^{\prime \prime}\right)} e^{2 \pi i z(x-y)} d z .
$$

Далее по (17) имеем

$$
\int \gamma\left(y+s-\omega^{\prime \prime}\right) e^{2 \pi i(x-z-s)} d y=c \frac{1}{\gamma\left(z-x+s+\omega^{\prime \prime}\right)},
$$

так что множители $\gamma\left(s-x+\omega^{\prime \prime}-z\right)$ сокращаются. В результате

$$
I(x, s)=c^{2} c(s) \frac{e^{2 \pi i s^{2}}}{\gamma\left(s-x+\omega^{\prime \prime}\right)} \int \frac{e^{2 \pi i z(x+s)}}{\gamma\left(z+\omega^{\prime \prime}\right)} d z=c^{2} e^{2 \pi i s^{2}} \psi(x, s),
$$

если использовать (16). Итак, функция Кашаева $\psi(x, s)$ является собственной функцией оператора $K^{-1}$ с собственным значением $c^{2} e^{2 \pi i s^{2}}$. Постоянный множитель $c^{2}$ можно опустить при описании оператора эволюции, которая в нашем представлении - просто умножение на $e^{-2 \pi i s^{2}}$, так что

$$
K^{-1} Z K=Z, \quad K^{-1} r K=e^{2 \pi i s^{2}} e^{-2 \pi i\left(s+\omega^{\prime}\right)^{2}} r=q^{1 / 2} e^{i \pi s / \omega} r=q^{1 / 2} Z r .
$$

Именно эта эволюция была получена в [4].

\section{§6. Заключение}

Итак, мы показали, что естественное включение нулевых мод в матрицу монодромии оператора $\widehat{L}_{1}$ дает описание гильбертова пространства и эволюции для квазимомента $P$. 


\section{§7. Приложение}

Свойства модулярного квантового дилогарифма подробно описаны в статье [13] (а также во многих других местах). В нашей работе мы используем помимо функционального уравнения (7) следующие свойства.

Соотношения отражения

$$
\gamma(z) \gamma(-z)=\beta e^{i \pi z^{2}}, \quad \beta=e^{(i \pi / 12)(\tau+1 / \tau)} .
$$

Положение первого полюса

$$
\gamma\left(z+\omega^{\prime \prime}\right)=\frac{c}{z}, \quad c=-\frac{2}{2 \pi i \beta} e^{-i \pi / 4} .
$$

«Вещественность»

$$
\overline{\gamma(z)}=\frac{1}{\gamma(\bar{z})}
$$

Асимптотика

$$
\gamma(z) \rightarrow 1
$$

в секторе $-\pi / 4<\arg z<\pi / 4$.

Интегральное тождество

$$
\frac{\gamma(t+a)}{\gamma(t+b)}=\frac{c}{\gamma\left(b-a-\omega^{\prime \prime}\right)} \int \frac{\gamma\left(b-a-\omega^{\prime \prime}+z\right)}{\gamma\left(z+\omega^{\prime \prime}\right)} e^{2 \pi i z\left(t+a+\omega^{\prime \prime}\right)} d z,
$$

предел при $b \rightarrow \infty$

$$
\gamma(t+a)=c \int \frac{e^{2 \pi i z\left(t+a+\omega^{\prime \prime}\right)}}{\gamma\left(z+\omega^{\prime \prime}\right)} d z
$$

и его обращение

$$
\int \gamma(t+a) e^{-2 \pi i t s} d t=c \frac{e^{2 \pi i s\left(a+\omega^{\prime \prime}\right)}}{\gamma\left(s+\omega^{\prime \prime}\right)} .
$$

\section{ЛитературА}

[1] J.-L. Gervais, J. Schnittger, The many faces of the quantum Liouville exponentials, Nuclear Phys. B, 413 (1994), 433-457; http://arxiv.org/abs/hep-th/9308134.

[2] J. Teschner, A Lecture on the Liouville vertex operators, Internat. J. Modern. Phys. A, 19 (2004), May, suppl., 436-458; http://arxiv.org/abs/hep-th/0303150.

[3] O. Babelon, Universal exchange algebra for Bloch waves and Liouville theory, Comm. Math. Phys., 139:3 (1991), 619-643.

[4] L. D. Faddeev, A. Y. Volkov, Discrete evolution for the zero-modes of the quantum Liouville model, J. Phys. A, 41 (2008), no. 19, 194008; http://arxiv.org/abs/ 0803.0230 .

[5] L. D. Faddeev, L. A. Takhtajan, Liouville model on the lattice, in: Lecture Notes in Phys., vol. 246, Springer-Verlag, Berlin, 1986, 166-179.

[6] В. Г. Дринфельд, Алгебры Хопфа и квантовое уравнение Янга-Бакстера, Докл. АН СССР, сер. физ., 283:5 (1985), 1060-1064.

[7] Л. Д. Фаддеев, Н. Ю. Решетихин, Л. А. Тахтаджян, Квантование групп Ли и алгебр Ли, Алгебра и анализ, 1 (1989), 178-206.

[8] R. M. Kashaev, On the spectrum of Dehn twists in quantum Teichmuller theory, http://arxiv.org/abs/math/0008148.

[9] В. В. Фок, Л. О. Чехов, Квантовые пространства Тейхмюллера, ТМФ, 120:3 (1999), 511-528; http://arxiv.org/abs/math/9908165. 
[10] S. E. Derkachov, L. D. Faddeev, 3j-symbol for the modular double of $S L_{q}(2, R)$ revisited, http://arxiv.org/abs/1302.5400.

[11] R. M. Kashaev, The quantum dilagarithm and Dehn twists in quantum Teichmuller theory, in: Integrable structures of Exactly Solvable Two-Dimansional Models of Quantum Field Theory (Kiev, 2000), NATO Sci. Ser. II Math. Phys. Chem., vol. 35, Kluwer Acad. Publ., Dordrecht, 2001, 211-221.

[12] L. D. Faddeev, Discrete Heisenberg-Weyl group and modular group, Lett. Math. Phys., 34:3 (1995), 249-254; http://arxiv.org/abs/hep-th/9504111.

[13] A. Y. Volkov, Noncommutative hypergeometry, Comm. Math. Phys., $258: 2$ (2005), 257-273; http://arxiv.org/abs/math/0312084.

[14] A. B. Zamolodchikov, A. B. Zamolodchikov, Conformal bootstrap in Liouville field theory, Nuclear Phys. B, 477:2 (1996), 577-605; http://arxiv.org/abs/ hep-th/9506136.

[15] G. Jorjadze, G. Weigt, Zero mode problem of Liouville field theory, http: //arxiv .org/ $\mathrm{abs} / \mathrm{hep}-\mathrm{th} / 0207041$.

Санкт-Петербургское отделение

Математического института им. В. А. Стеклова РАН, Санкт-Петербургский государственный университет e-mail: faddeev@pdmi.ras.ru
Поступило в редакцию 13 января 2014 г. 